Authorship note: LFB and IZ contributed equally to this work.

Conflict of interest: DK is Founder of Lysosomal Therapeutics Inc. and Vanqua Bio; serves on the scientific advisory boards of The Silverstein Foundation for Parkinson's with CBA, Intellia Therapeutics, and Prevail Therapeutics; and is a venture partner at OrbiMed. PB has consulted for Axial Therapeutics, Calico, CuraSen, Fujifilm Cellular Dynamics Inc., Idorsia, IOS Press Partners, Lundbeck, and Living Cell Technologies. He has received commercial support for research from Lundbeck and Roche. He has ownership interests in AcouSort and Axial Therapeutics and is on the steering committee of the Nilotinib in Parkinson's Disease trial.

Copyright: ( 2021 , Burbulla et al. This is an open access article published under the terms of the Creative Commons Attribution 4.0 International License.

Submitted: February 15, 2021

Accepted: August 25, 2021

Published: October 8, 2021

Reference information: JCI Insight. 2021;6(19):e148649.

https://doi.org/10.1172/jici. insight.148649.

\section{Direct targeting of wild-type glucocerebrosidase by antipsychotic quetiapine improves pathogenic phenotypes in Parkinson's disease models}

\author{
Lena F. Burbulla, ${ }^{1,2,3,4}$ Jianbin Zheng, ${ }^{1,5}$ Pingping Song, ${ }^{1}$ Weilan Jiang, ${ }^{1}$ Michaela E. Johnson, ${ }^{6}$ \\ Patrik Brundin, ${ }^{6}$ and Dimitri Krainc ${ }^{1}$ \\ 1Department of Neurology, Northwestern University Feinberg School of Medicine, Chicago, Illinois, USA. ${ }^{2}$ German Center \\ for Neurodegenerative Diseases (DZNE), Munich, Germany. ${ }^{3}$ Metabolic Biochemistry, Biomedical Center (BMC), Faculty \\ of Medicine, Ludwig Maximilians University, Munich, Germany. ${ }^{4}$ Munich Cluster for Systems Neurology (SyNergy), Munich, \\ Cermany. ${ }^{5}$ Department of Chemistry, Chemistry of Life Processes Institute, Center for Molecular Innovation and Drug \\ Discovery, and Center for Developmental Therapeutics, Northwestern University, Evanston, Illinois, USA. ${ }^{6}$ Parkinson's \\ Disease Center, Department of Neurodegenerative Science, Van Andel Institute, Grand Rapids, Michigan, USA.
}

\begin{abstract}
Current treatments for Parkinson's disease (PD) provide only symptomatic relief, with no diseasemodifying therapies identified to date. Repurposing FDA-approved drugs to treat PD could significantly shorten the time needed for and reduce the costs of drug development compared with conventional approaches. We developed an efficient strategy to screen for modulators of $\beta$-glucocerebrosidase (GCase), a lysosomal enzyme that exhibits decreased activity in patients with $\mathrm{PD}$, leading to accumulation of the substrate glucosylceramide and oxidized dopamine and $\alpha$-synuclein, which contribute to PD pathogenesis. Using a CCase fluorescent probe and affinitybased fluorescence polarization assay, we screened 1280 structurally diverse, bioactive, and cell-permeable FDA-approved drugs and found that the antipsychotic quetiapine bound GCase with high affinity. Moreover, quetiapine treatment of induced pluripotent stem cell-derived (iPSC-derived) dopaminergic neurons from patients carrying mutations in GBA1 or LRRK2 led to increased wild-type GCase protein levels and activity and partially lowered accumulation of oxidized dopamine, glucosylceramide, and $\alpha$-synuclein. Similarly, quetiapine led to activation of wild-type GCase and reduction of $\alpha$-synuclein in a GBA mutant mouse model (Gba ${ }^{0409 \mathrm{~V} /++}$ mice). Together, these results suggest that repurposing quetiapine as a modulator of GCase may be beneficial for patients with PD exhibiting decreased GCase activity.
\end{abstract}

\section{Introduction}

Drug repurposing has the potential to generate urgently needed disease-modifying treatment for Parkinson's disease (PD), the second most common neurodegenerative disease (1), more quickly and in a more cost-effective manner compared with traditional de novo drug discovery. PD is pathologically defined by the presence of intracellular proteinaceous inclusions, called Lewy bodies, primarily composed of insoluble aggregates of $\alpha$-synuclein and the loss of dopaminergic neurons in the substantia nigra $(2,3)$. Variants in the GBA1 gene encoding the lysosomal enzyme $\beta$-glucocerebrosidase (GCase) represent the most common genetic risk factor for PD (4). Loss-of-function GBA1 mutations lead to the accumulation of its substrate glucosylceramide (GluCer) and $\alpha$-synuclein, contributing to a toxic pathogenic cascade (5). We and others have previously shown that decreased wild-type GCase activity might also contribute to the pathogenesis in genetic and sporadic PD forms that are not linked to GBA1 mutations (6-12). This suggests that activating the wild-type enzyme might be a viable therapeutic approach. We recently showed that modulation of wildtype GCase with a novel small molecule increased the enzyme activity and partially ameliorated pathological phenotypes in dopaminergic neurons from patients with GBA1-linked and non-GBA1-linked PD (13).

In this work, we used a fluorescent probe based on the modification of a GCase small molecule analogue (14) and screened FDA-approved drugs for their capacity to bind GCase in a fluorescence polarization (FP) 
high-throughput screen (HTS). We identified quetiapine, an antipsychotic commonly used to treat patients with dementia and psychosis (15), as the CNS drug with the greatest binding affinity. Moreover, we found that quetiapine increased wild-type GCase protein levels and activity and partially ameliorated dopamine oxidation and $\alpha$-synuclein accumulation in iPSC-derived dopaminergic neurons from patients with GBA1-linked and LRRK2-linked PD. Administration of quetiapine to GBA mutant mice led to activation of GCase in the $\mathrm{CNS}$ and reduced $\alpha$-synuclein accumulation. These results raise the possibility that quetiapine may modulate GCase activity and thereby slow disease progression in PD.

\section{Results}

Affinity-based FP screen of FDA-approved drugs identifies quetiapine as a binder of GCase. FP is a powerful technique for the nondisruptive measuring of biomolecular interactions in solution. One important application of FP-based assays is the FP competition assay to screen for small-molecule compounds that compete FP probe-protein interactions $(16,17)$. The principle of this assay relies on an increase of FP response once the fluorescent small-molecule probe is noncovalently bound to the protein of interest. Equilibrium displacement of the protein-bound probe by a test compound results in reduced FP due to the faster rotation of the unbound probe (16). To facilitate the discovery of noninhibitory GCase modulators, we designed the FP probe, JZ-3165 (Figure 1A), by linking a fluorescent probe (TAMRA) to an optimized binding moiety. The latter was based on a structure-activity relationship study of the GCase compound with a short polyethylene glycol linker. JZ-3165 exhibited a dissociation constant $\left(K_{D}\right)$ value of $0.71 \mu \mathrm{M}$ binding affinity with recombinant GCase. Using this probe, we developed an affinity-based FP HTS to screen 1280 structurally diverse, bioactive, and cell-permeable compounds approved by the FDA. We identified a group of drugs that were able to bind GCase with varying affinity (Supplemental Table 1; supplemental material available online with this article; https://doi. org/10.1172/jci.insight.148649DS1), of which the CNS drug quetiapine (Figure 1B) showed the greatest affinity, with a $K_{D}$ value of $5 \mu \mathrm{M}$ (Figure 1C). Among the identified hits, perphenazine and fluphenazine had similar chemical structures as quetiapine, indicating that they may share the same binding site on GCase, but they exhibited higher $K_{D}$ values, making them less likely to affect GCase levels and activity.

Quetiapine improves pathogenic phenotypes in iPSC-derived dopaminergic neurons from patients with GBA1-linked $P D$. To determine whether quetiapine could modulate wild-type GCase levels and activity in human neurons, we examined iPSC-derived dopaminergic neurons from a PD patient line harboring the heterozygous 84GG GBA1 mutation (c.84dupG frame-shift mutation) that results in complete loss of mutant GCase and reduced wild-type protein from a single copy of the wild-type GCase allele and used a CRISPR-corrected isogenic line as control. We found reduced GCase protein levels and activity in the GBA1 patient line compared with those in an isogenic control line, which were partially corrected by treatment with increasing concentrations of quetiapine (Figure 2, A and B). Next, we examined whether increasing wild-type GCase levels and activity were sufficient to modify disease-related pathogenic phenotypes in patient neurons. Treatment with quetiapine partially reduced the accumulation of intracellular total GluCer species (Figure 2C) and lowered $\alpha$-synuclein levels in the Triton-soluble fraction (Figure 2D), whereas levels of glucosylsphingosine and Triton-insoluble $\alpha$-synuclein were not changed (Supplemental Figure 1). We have recently reported that dopaminergic neurons from patients with familial or sporadic PD exhibit accumulation of oxidized dopamine that contributes to lysosomal dysfunction $(6,13)$. Interestingly, increasing concentrations of quetiapine partially ameliorated accumulation of oxidized dopamine in neurons from patients with GBA1 PD compared with isogenic control neurons (Figure 2E and Supplemental Figure 2).

Quetiapine treatment leads to partial rescue of pathogenic phenotypes in LRRK2-linked PD iPSC-derived dopaminergic neurons. Previous reports have shown that wild-type GCase activity is reduced in brain tissue and iPSC-derived neurons from patients with genetic and sporadic forms of PD (6-11), suggesting that improving wildtype GCase activity may prove beneficial. Here, we sought to investigate whether treatment with quetiapine would be sufficient to increase GCase levels and activity in mutant LRRK2 G2019S PD patient lines that exhibit decreased activity of wild-type GCase (18). Treatment with quetiapine led to an increase in GCase protein levels (Figure 3A) as well as enzyme activity (Figure 3B) and partially reduced the accumulation of $\alpha$-synuclein in the Triton-soluble (Figure 3C) as well as Triton-insoluble fraction (Supplemental Figure 3) in neurons from patients with LRRK2 PD (LRRK2-PD1). We previously found accumulation of oxidized dopamine in dopaminergic neurons from patients with various forms of non-GBA1-linked PD, including LRRK2 $(6,11,13)$. Importantly, treatment of neurons from patients with LRRK2 PD with quetiapine led to decreased levels of toxic oxidized dopamine (Figure 3D). These findings were confirmed in another LRRK2 
A

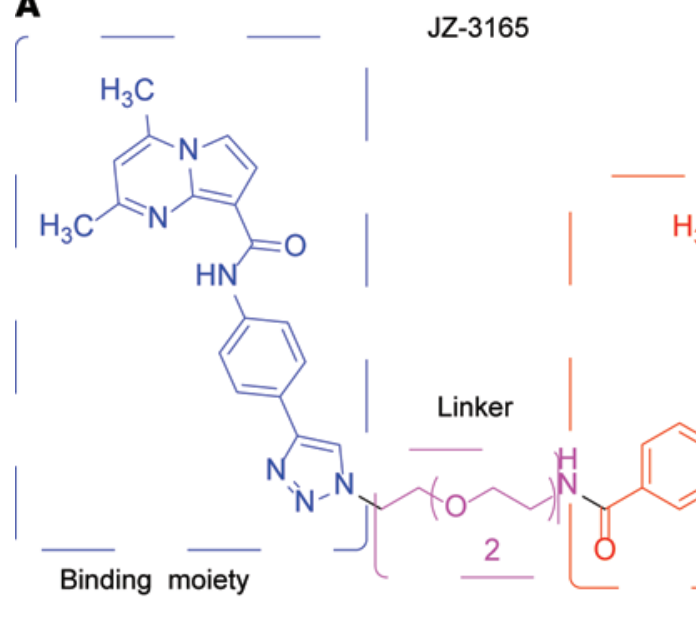

\section{C}

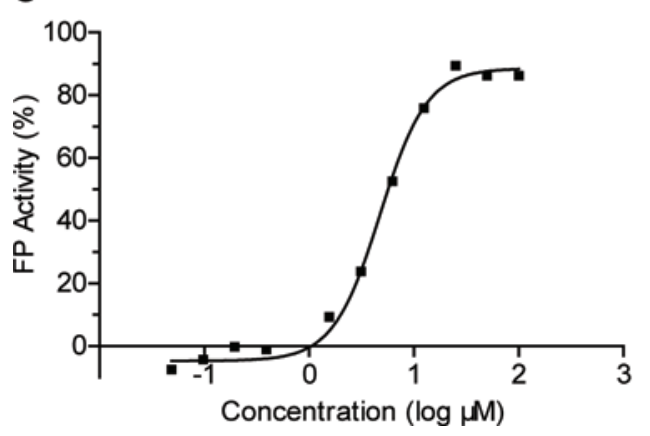

B

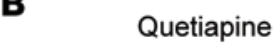

TAMRA<smiles></smiles>

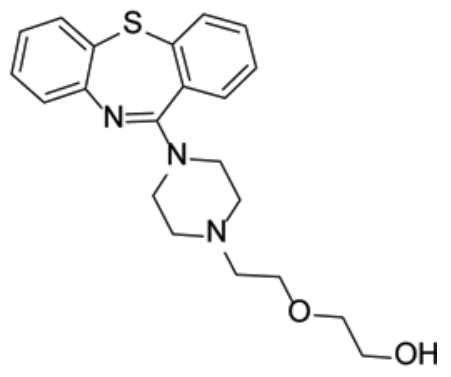

Figure 1. Affinity-based fluorescence polarization screen of FDA-approved drugs identifies quetiapine as a GCase binder. (A and B) Chemical structures of (A) fluorescence polarization (FP) probe JZ-3165 and (B) quetiapine. (C) Quetiapine FP activity in the FP assay using probe JZ-3165.

G2019S PD patient line (LRRK2-PD2), whereby an increase of GCase activity upon quetiapine treatment resulted in the reduction of oxidized DA (Supplemental Figure 4).

Wild-type GCase activation by quetiapine treatment in mice lowers $\alpha$-synuclein accumulation. To determine whether quetiapine can modulate GCase activity in vivo, it was administered, via intraperitoneal injections, at $5 \mathrm{mg} / \mathrm{kg}$ to $\mathrm{Gba1} 1^{\mathrm{D} 409 \mathrm{~V} /+}$ mutant mice twice daily for 15 days. This led to an increase in GCase activity in brain tissue from mice treated with quetiapine compared with that in vehicle-treated mice (Figure $4 \mathrm{~A}$ ). Activation of GCase with quetiapine also reduced Triton-insoluble $\alpha$-synuclein (19) in brain tissues of $G b a 1^{\text {D409V/+ }}$ mutant mice (Figure 4B), whereas Triton-soluble $\alpha$-synuclein was not changed (Supplemental Figure 5). These results suggest that quetiapine can modify GCase activity in vivo and has beneficial effects on reducing $\alpha$-synuclein.

\section{Discussion}

Our results suggest that increasing wild-type GCase activity could serve as a potential therapeutic approach for various forms of PD that exhibit decreased GCase activity. Decreased GCase activity contributes to PD pathogenesis not only in patients who carry GBA1 mutations, but also in patients with sporadic or other genetic forms of PD who do not harbor GBA1 mutations (6-12). We have previously described that GCase and $\alpha$-synuclein form a positive feedback loop, in which $\alpha$-synuclein inhibits trafficking of wild-type or mutant GCase to lysosomes, leading to decreased lysosomal GCase activity that in turn contributes to $\alpha$-synuclein accumulation (5). In addition, we found that vulnerable midbrain neurons in multiple forms of PD accumulate toxic oxidized dopamine that modifies key cysteine residues in the catalytic site of wild-type GCase, further decreasing GCase activity (6). Previous screens for GCase modulators measured enzyme activity using artificial substrates that are not physiologically relevant, such as 4-methylumbelliferyl- $\beta$-D-glucopyranoside and resorufin $\beta$-D-glucopyranoside, and identified hit compounds were not validated with GluCer-like substrates (14). Using FP HTS and a unique probe, in this study we were able to identify quetiapine as a potentially novel GCase modulator that also ameliorates key pathogenic effects linked to deficient GCase activity in dopaminergic neurons. It will be of interest to examine the effects of quetiapine treatment in other genetic and sporadic forms of PD that exhibit decreased activity of wild-type GCase. 
A
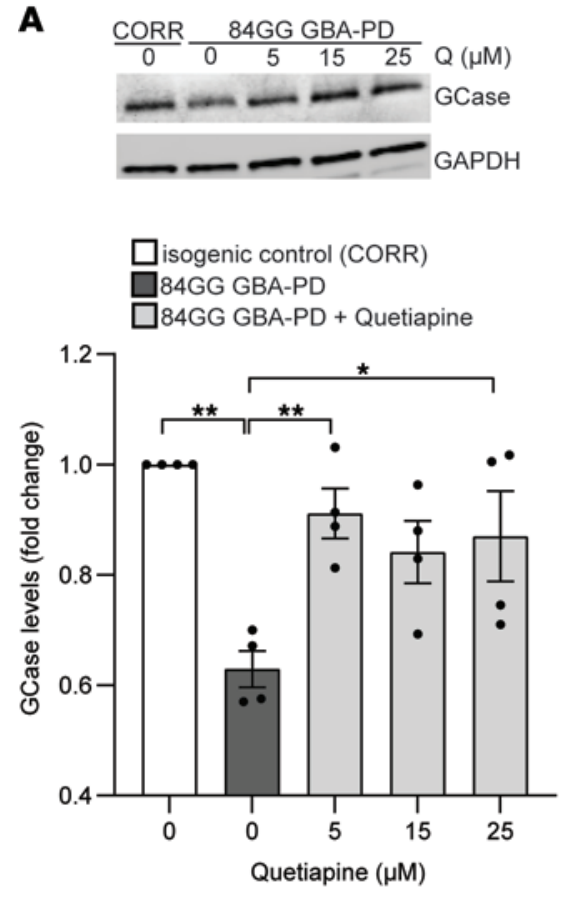

D
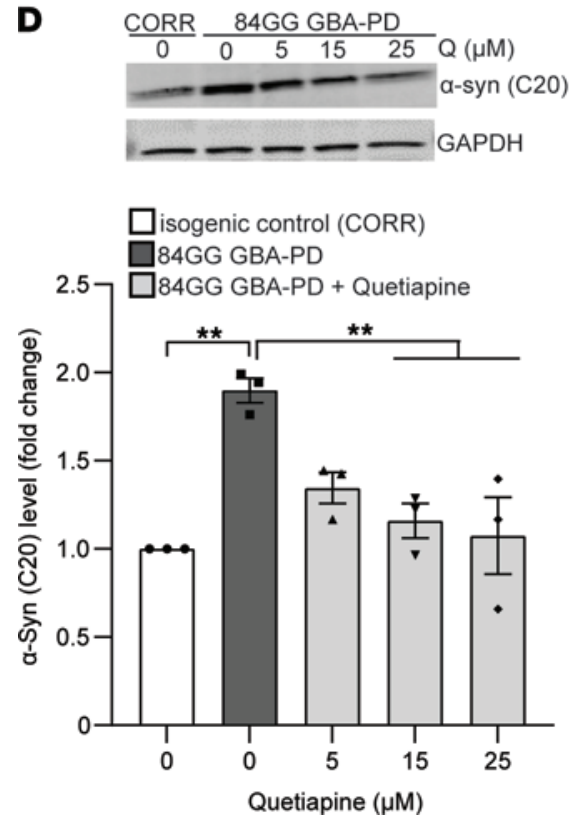
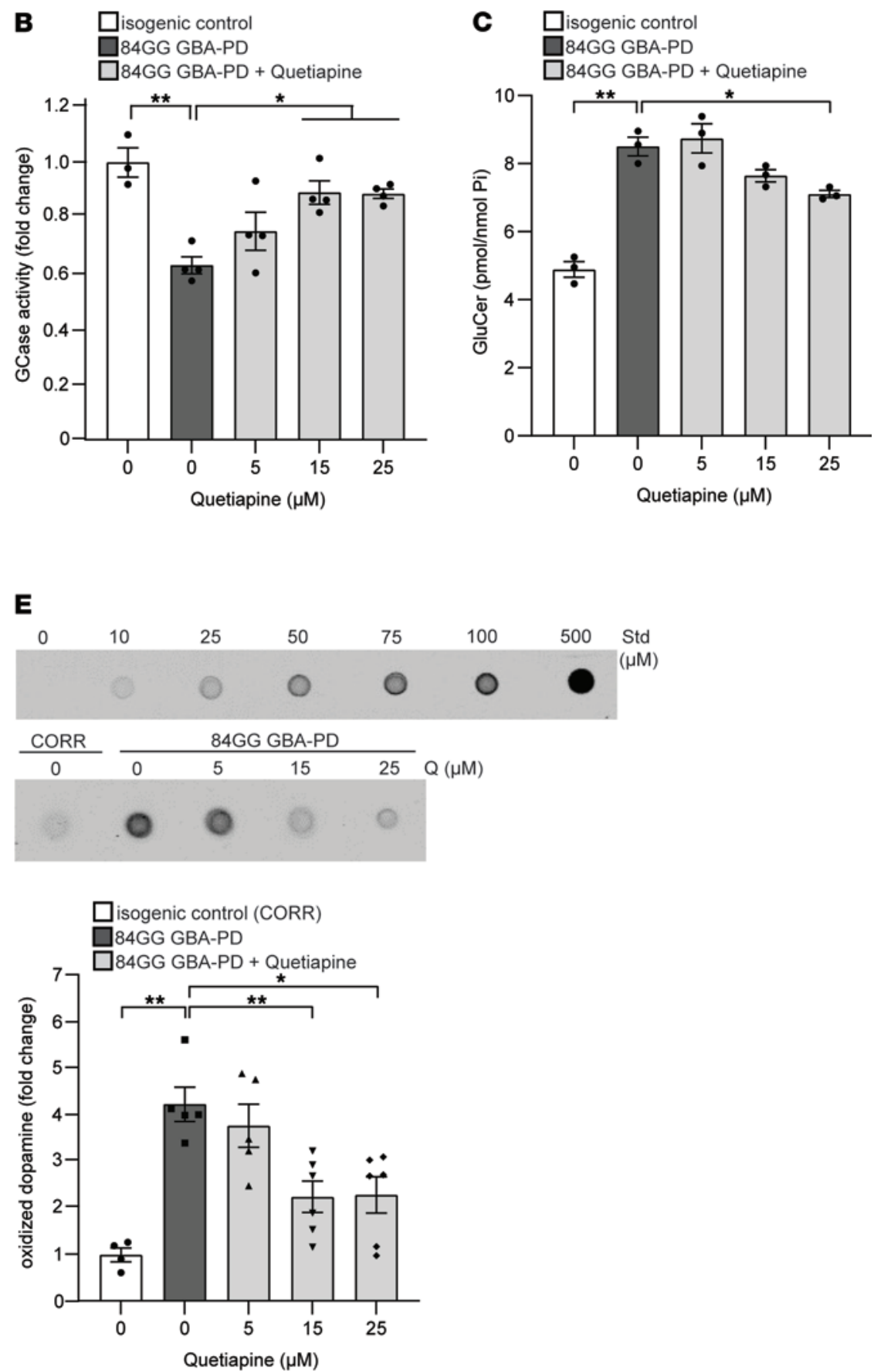

Figure 2. Quetiapine increases wild-type $\beta$-glucocerebrosidase and lowers pathogenic phenotypes in GBA1-linked iPSC-derived dopaminergic neurons. (A and B) Heterozygous 84GG CBA1 mutant dopaminergic neurons (84CG CBA-PD) and isogenic control neurons with CBA1 mutation corrected by CRISPR/Cas9 gene editing (CORR) were treated with DMSO (vehicle) or quetiapine $(5,15$, and $25 \mu \mathrm{M})$ for 10 days. All samples were collected at day 130 of differentiation. Tritonsoluble lysates were analyzed for (A) GCase protein by immunoblotting ( $n=4$ independent experiments) and (B) GCase activity by in vitro enzyme activity assay ( $n=3-4$ independent experiments). (C) Quantification of intracellular total glucosylceramide (CluCer) species by mass spectrometry normalized to internal phosphate ( $\mathrm{Pi}$ ) ( $n=3$ independent experiments). (D) Immunoblot analysis of $\alpha$-synuclein in Triton-soluble lysates ( $n=3$ independent experiments). (E) Detection and quantification of oxidized dopamine (DA) performed by near-infrared fluorescence assay ( $n=4-6$ independent experiments). Standard of oxidized DA ranging from 0 to $500 \mu \mathrm{M}$ is shown. Error bars represent mean $\pm \mathrm{SEM}$. ${ }^{*} P<0.05,{ }^{* *} P<0.01,1$-way ANOVA with Tukey's post hoc test. Std, standard; Q, quetiapine.

Quetiapine was developed in 1985, was approved for medical use in the United States in 1997, and is now a commonly prescribed CNS drug, primarily for treatment of bipolar disorder, depression, and schizophrenia (20). Quetiapine is both effective and well tolerated, with fewer side effects than other antipsychotics, especially in patients with dementia who also exhibit psychosis (21). Repurposing strategies of currently approved and previously studied drugs facilitate speeding up of the traditional process of drug discovery by 
A
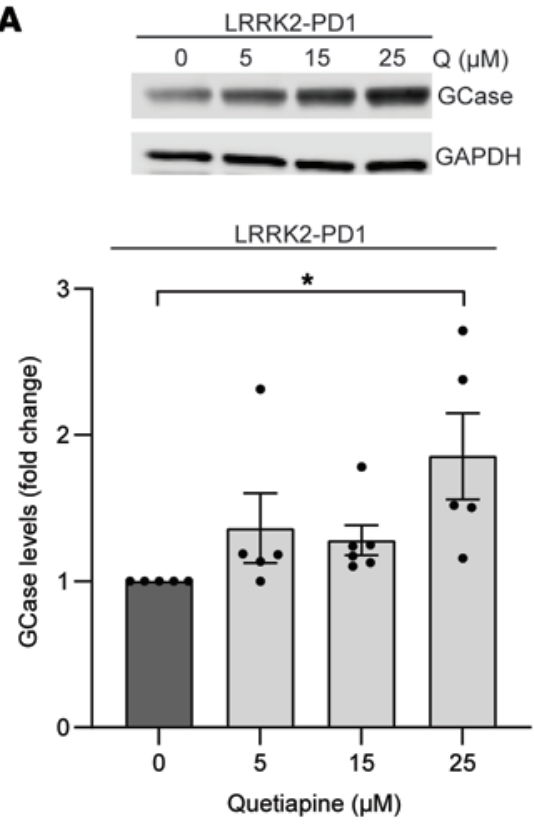

B
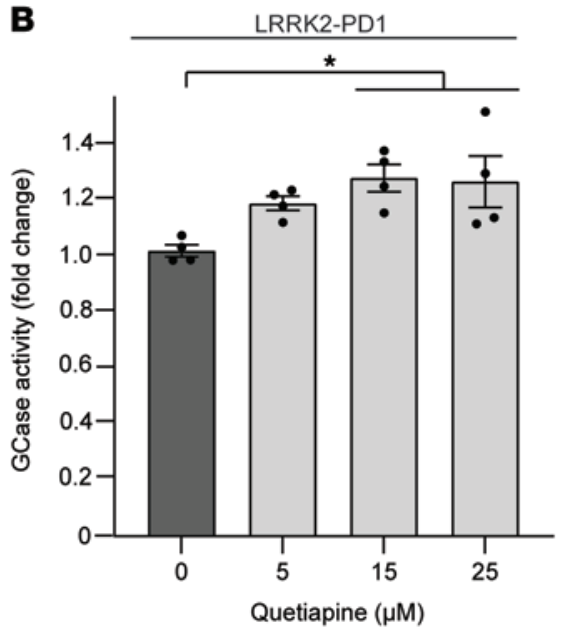

C
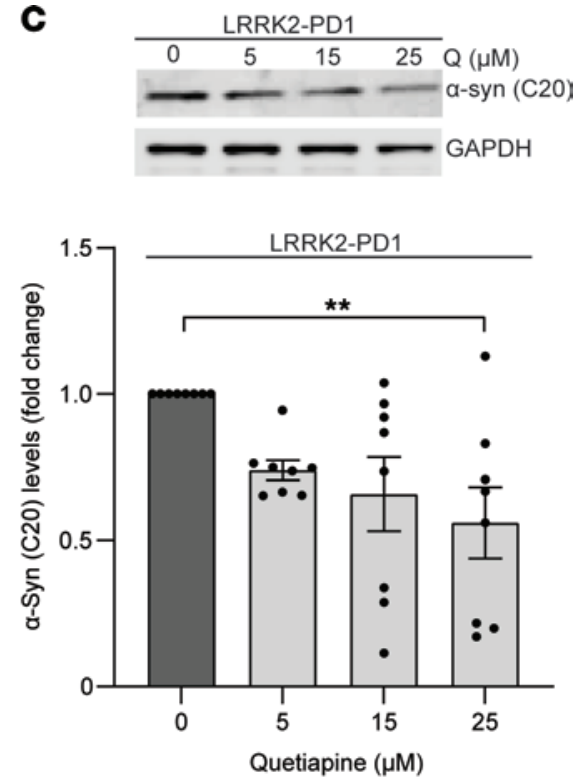

D
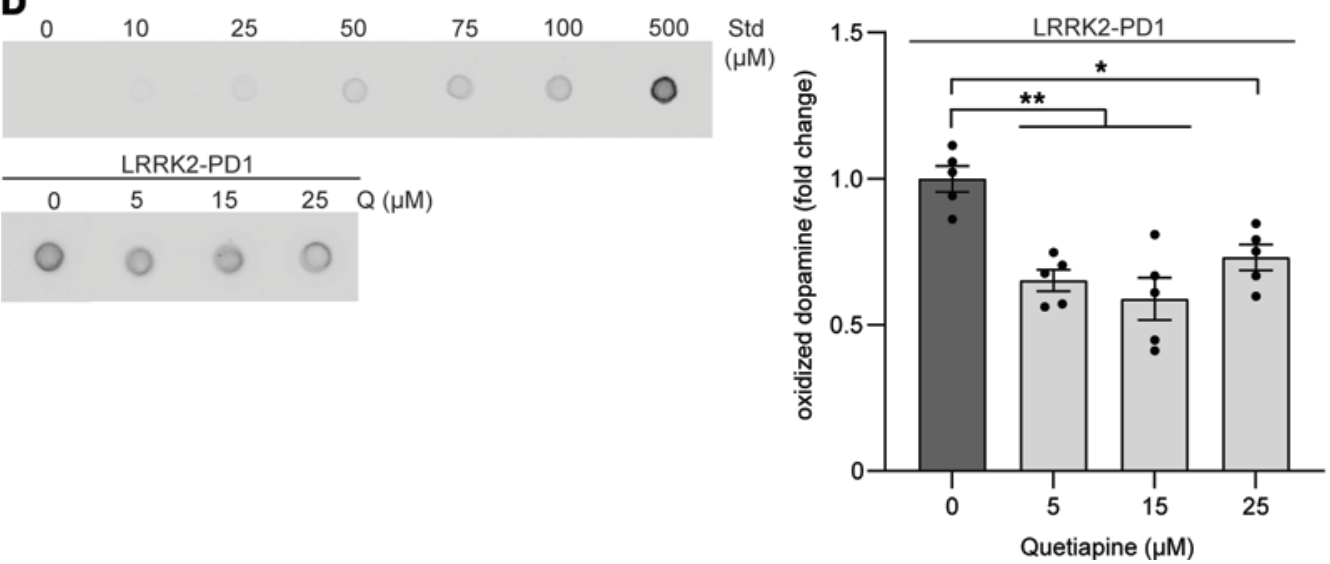

Figure 3. Quetiapine treatment leads to wild-type $\beta$-glucocerebrosidase activation and partial rescue of pathogenic phenotypes in non-CBA1-linked PD iPSC-derived dopaminergic neurons. LRRK2 G2019S mutant dopaminergic neurons (LRRK2-PD1) were treated with DMSO (vehicle) or quetiapine (5, 15, and $25 \mu \mathrm{M}$ ) for 10 days. All samples were collected at day 100 of differentiation. (A and B) Triton-soluble lysates were analyzed for (A) GCase protein by immunoblotting ( $n=5-6$ independent experiments) and (B) GCase activity by in vitro enzyme activity assay ( $n=4$ independent experiments). (C) Immunoblot analysis of $\alpha$-synuclein in Triton-soluble lysates ( $n=8$ independent experiments). (D) Cell lysates were analyzed for oxidized dopamine (DA) by near-infrared fluorescence assay ( $n=5$ independent experiments). Standard of oxidized DA ranging from 0 to $500 \mu \mathrm{M}$ is shown. Error bars represent mean \pm SEM. ${ }^{*} P<$ $0.05,{ }^{* *} P<0.01,1$-way ANOVA with Tukey's post hoc test. Std, standard; Q, quetiapine.

identifying a clinical use for drugs that have already proved to be safe and effective in humans. This strategy, which is also actively explored in PD (22), may also lower overall costs and shorten development timelines (23). Our findings highlight the potential value of quetiapine as a therapeutic approach for different forms of PD, including familial and sporadic PD, which exhibit decreased wild-type GCase activity. Future clinical studies will be required to fully evaluate quetiapine's potential for the treatment of PD and to define appropriate doses for long-term therapy.

\section{Methods}

Cell-free enzyme activity assay. Cell-free in vitro enzyme activity assay was performed as described previously (24). The recombinant GCase enzyme velaglucerase alfa (Vpriv, Shire Human Genetic Therapies Inc.) was obtained from residual solution after clinical infusions.

Determination of probe (JZ-3165) binding affinity by FP assay. Synthesis of FP probe JZ-3165 has previously been reported (25). JZ-3165 ( $25 \mathrm{~nL} /$ well, $50 \mathrm{nM}$ final concentration) was transferred into 384-well black plates 
A

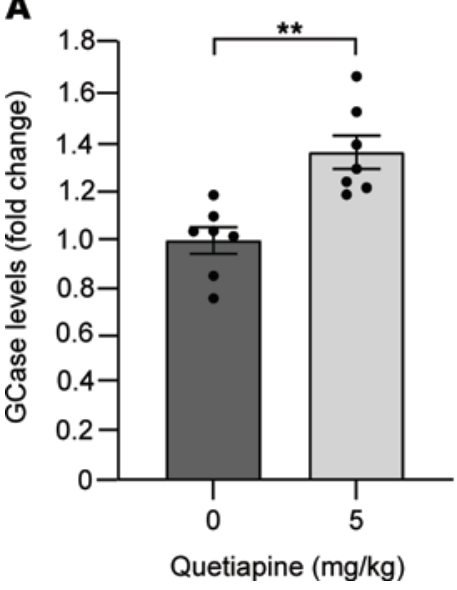

B

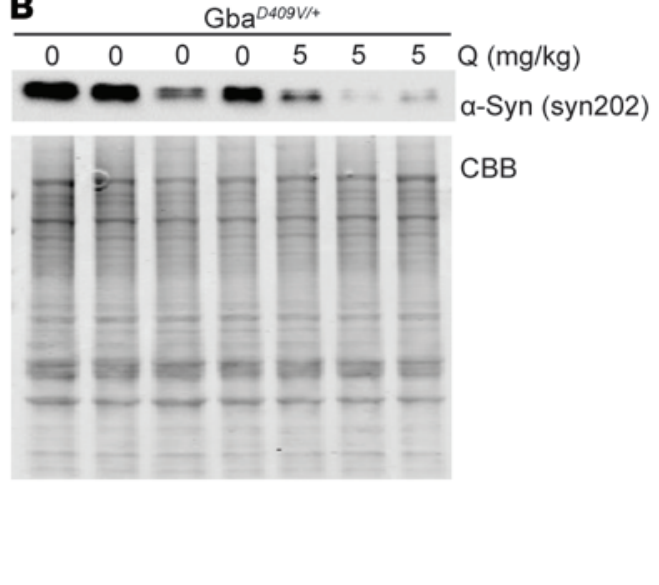

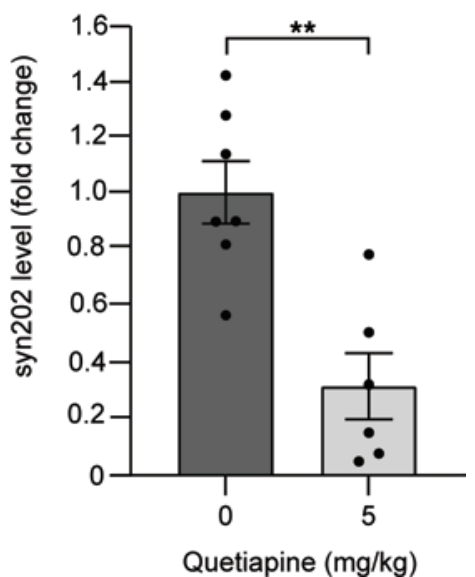

Figure 4. Wild-type $\beta$-glucocerebrosidase activation by quetiapine treatment in mice lowers $\alpha$-synuclein accumulation. Gba $1^{0409 V /+}$ mutant mice were treated with saline (vehicle) or quetiapine $(5 \mathrm{mg} / \mathrm{kg}$ ) intraperitoneally twice daily for 15 days. (A) $\beta$-Glucocerebrosidase (GCase) activity was measured by in vitro enzyme activity assay in Triton-soluble lysates of hippocampal tissue ( $n=7$ saline- and $n=7$ quetiapine-treated mice) (B) Immunoblot analysis of $\alpha$-synuclein in Triton-insoluble lysates of hippocampal tissue ( $n=7$ saline- and $n=6$ quetiapine-treated mice). Coomassie Brilliant blue (CBB) was used as a loading control. Error bars represent mean \pm SEM. ${ }^{* *} P<0.01$, Student's $t$ test. Q, quetiapine.

using the Labcyte Echo 550 Liquid Handler system. $25 \mu \mathrm{L}$ /well enzyme dilutions in GCase activity assay buffer (final titration: $5 \mathrm{nM}$ to $10 \mu \mathrm{M}, 10$ concentrations, 2-fold dilution) were added. A buffer solution without GCase protein was used as a blank control. The plate was shaken at room temperature in the dark for 20 minutes. FP was measured using a Tecan Infinity M1000 microplate reader $(E x=535 \mathrm{~nm}$ and $E m=580 \mathrm{~nm})$.

In vitro FP HTS using JZ-3165 as a probe. A mixture of GCase protein $(2 \mu \mathrm{M})$ and JZ-3165 probe $(50 \mathrm{nM})$ in GCase enzyme activity buffer $(2 \mu \mathrm{L} /$ well $)$ was added into a NUNC black 1536 -well plate. A single dose $(5 \mathrm{~nL}$, $25 \mu \mathrm{M}$ final concentration) of drugs in DMSO (10 mM) solution was transferred into the plate, incubated for 20 minutes, and read at established conditions. Activities were confirmed by dose-response curves using freshly prepared solutions from solid samples.

Generation of human iPSCs and differentiation into midbrain dopaminergic neurons. Both iPSC lines from PD patients with mutations in GBA1 were obtained from Northwestern University Biorepository (13). The 2 iPSC lines from PD patients with G2019S LRRK2 mutations were obtained from the National Institute of Neurological Disorders and Stroke Repository at the Coriell Institute for Medical Research (catalog no. ND29423 and ND29492).

iPSCs were maintained in mTeSR1 (StemCell Technologies) on Matrigel-coated (Corning) plates. Dopaminergic neuron differentiation was performed according to published protocols (26) and as previously described (6). Neural growth factors were removed on day 40, and cells were maintained in Neurobasal Media (Thermo Fisher Scientific, 21103-049) with NeuroCult SM1 supplement (STEMCELL Technologies, 5711). All iPSC lines were found to differentiate at similar efficiencies. Results were obtained from a set of at least 3 independent dopaminergic neuron differentiations. All iPSC lines were routinely tested for mycoplasma contamination.

Gba1 $1^{\text {D409V/+ }}$ mice. Gba1 ${ }^{\text {D409V/+ }}$ (C57BL/6N-GBAtm1.1Mjff) mice (The Jackson Laboratory) were bred following guidelines from the Institutional Animal Care and Use Committee at Northwestern University and handled in accordance with the US NIH Guide for the Care and Use of Laboratory Animals (National Academies Press, 2011) and Society for Neuroscience guidelines. Mice (mixed group of male and female animals) aged $17-19$ months received saline or quetiapine $(5 \mathrm{mg} / \mathrm{kg}$ ) through intraperitoneal injection, twice per day, 5 days per week, over 3 weeks.

Sequential biochemical extraction. Sequential biochemical extraction of proteins from iPSC-derived neurons and mouse tissue has been previously described (6). Neurons were harvested and centrifuged at $300 \mathrm{~g}$ for 5 minutes, and cell pellets were homogenized for extraction in 1\% Triton X-100 lysis buffer. After incubation on ice for 30 minutes, samples were centrifuged at 100,000 g for 30 minutes at $4^{\circ} \mathrm{C}$. Supernatant was analyzed as the Triton-soluble fraction. Insoluble pellets were extracted in 2\% SDS/50 mM Tris (pH 7.4) buffer and further centrifuged at $150,000 \mathrm{~g}$ for 30 minutes at $4^{\circ} \mathrm{C}$. SDS-insoluble pellets were further processed for near-infrared fluorescence assay. For biochemical extraction of brain tissue from mice, hippocampal brain tissue was homogenized in 1\% Triton X-100 lysis buffer according to tissue weight. Insoluble pellets from a 100,000g 
spin were extracted in $2 \%$ SDS $/ 50 \mathrm{mM}$ Tris ( $\mathrm{pH} 7.4$ ) by boiling and sonication. Samples were centrifuged at $150,000 \mathrm{~g}$ for 30 minutes at $4^{\circ} \mathrm{C}$, and the supernatant was analyzed as the SDS-soluble fraction.

Near-infrared fluorescence detection of oxidized dopamine. The near-infrared fluorescence assay was performed as described previously $(6,27)$. Leftover pellets from SDS extraction were extracted in $1 \mathrm{M} \mathrm{NaOH}$ at $55^{\circ} \mathrm{C}$ overnight. Then, samples were dried, washed with $\mathrm{H}_{2} \mathrm{O}$, lyophilized, and solubilized in $\mathrm{H}_{2} \mathrm{O}$. Samples and standard solutions from a $10 \mathrm{mM}$ oxidized dopamine stock were dropped on a Biodyne Nylon Transfer Membrane (Pall). Membranes were scanned using Odyssey infrared imaging system (LI-COR) with the 700 channel. Samples were quantified by obtaining integrated spot intensities using Odyssey infrared imaging software (LI-COR).

Antibodies. The following primary antibodies were used: rabbit anti-GCase (MilliporeSigma, G4171, 1:1000), $\alpha$-synuclein (C-20, Santa Cruz Biotechnology, SC-7011-R, 1:1000), $\alpha$-synuclein (syn202, BioLegend, MMS-529R, 1:1000), and GAPDH (MilliporeSigma, MAB374, 1:5000).

Assay for in vitro activity of GCase in neurons and mouse brain tissue. Activity was measured from Triton-soluble fractions of whole-cell neuronal lysates as described previously (10). Hippocampal brain tissue from mice was homogenized in 1\% Triton X-100 lysis buffer according to tissue weight and processed and analyzed as previously described (13).

Quantification of GluCer by SFC/MS/MS analysis. Neurons were harvested in cold PBS and centrifuged at $200 \mathrm{~g}$ for 5 minutes; cell pellets were rapidly stored at $-80^{\circ} \mathrm{C}$. Quantification of GluCer and glucosylsphingosine was performed as a service provided by the Lipidomics Core facility at the Medical University of South Carolina. Samples were normalized to total cellular phosphate (Pi) levels and expressed as picomole/nanomole Pi.

Statistics. Comparison of multiple groups was performed using 1-way ANOVA followed by Tukey's post hoc test; comparisons between 2 groups were performed using 2-tailed $t$ tests. $P$ values of less than 0.05 were considered significant. All data shown are representative of experiments from $n \geq 3$ independent experiments. All errors bars represent SEM.

Study approval. Animal studies were approved by the Northwestern University Animal Care and Use Committee.

\section{Author contributions}

DK was responsible for the overall direction of the project. LFB was responsible for Figures 2 and 3; Supplemental Figure 1A; Supplemental Figure 2, C, D, F, and G; and Supplemental Figure 4. JZ was responsible for Figure 1. PS was responsible for Figure 4; Supplemental Figure 1B; Supplemental Figure 3; and Supplemental Figure 5. WJ was responsible for Supplemental Figure 2, A, B, and E. JZ developed, performed, and analyzed the results of the FP HTS. MEJ and PB provided expert advice and critical review of the manuscript. LFB constructed the figures and analyzed the results shown in Figures $2-4$. LFB, JZ, and DK wrote the manuscript.

\section{Acknowledgments}

We thank Northwestern High Throughput Analysis Laboratory for FP HTS and Northwestern Stem Cell Core Facility for iPSC line generation from the GBA1 mutation carriers. We thank Ai-zhi Bai for assistance with FP HTS and Nicolas Marotta and Sohee Jeon for mouse breeding and genotyping. Lipid analyses were performed by the Lipidomics Shared Resource, Hollings Cancer Center, and Medical University of South Carolina. Mouse drug administration was performed by the Northwestern Developmental Therapeutics Core at Northwestern University. This work was supported by NIH grants R01NS076054 and R01NS096240 (to DK); by The Michael J. Fox Foundation for Parkinson's Research (13881 to JZ); by, in part, NIH core support grant P30 NS081774; and by the DFG (German Research Foundation) under Germany's Excellence Strategy, within the framework of the Munich Cluster of Systems Neurology (EXC 2145 SyNergy - ID 390857198).

Address correspondence to: Dimitri Krainc, Northwestern University Feinberg School of Medicine, 303 East Chicago Avenue, Ward 12-140, Chicago, Illinois 60611, USA. Phone: 312.503.3936; Email: dkrainc@nm.org.

1. Poewe W, et al. Parkinson disease. Nat Rev Dis Primers. 2017;3:17013.

2. Surmeier DJ, et al. Parkinson's disease is not simply a prion disorder. J Neurosci. 2017;37(41):9799-9807.

3. Spillantini MG, et al. Alpha-synuclein in Lewy bodies. Nature. 1997;388(6645):839-840.

4. Sidransky E, et al. Multicenter analysis of glucocerebrosidase mutations in Parkinson's disease. N Engl J Med. 2009;361(17):1651-1661.

5. Mazzulli JR, et al. Gaucher disease glucocerebrosidase and $\alpha$-synuclein form a bidirectional pathogenic loop in synucleinopathies. 
Cell. 2011;146(1):37-52.

6. Burbulla LF, et al. Dopamine oxidation mediates mitochondrial and lysosomal dysfunction in Parkinson's disease. Science. 2017;357(6357):1255-1261.

7. Gegg ME, et al. Glucocerebrosidase deficiency in substantia nigra of Parkinson disease brains. Ann Neurol. 2012;72(3):455-463.

8. Murphy KE, et al. Reduced glucocerebrosidase is associated with increased $\alpha$-synuclein in sporadic Parkinson's disease. Brain. 2014;137(Pt 3):834-848.

9. Chiasserini D, et al. Selective loss of glucocerebrosidase activity in sporadic Parkinson's disease and dementia with Lewy bodies. Mol Neurodegener. 2015;10:15

10. Mazzulli JR, et al. Activation of $\beta$-glucocerebrosidase reduces pathological $\alpha$-synuclein and restores lysosomal function in Parkinson's patient midbrain neurons. J Neurosci. 2016;36(29):7693-7706.

11. Nguyen M, Krainc D. LRRK2 phosphorylation of auxilin mediates synaptic defects in dopaminergic neurons from patients with Parkinson's disease. Proc Natl Acad Sci U S A. 2018;115(21):5576-5581.

12. Rocha EM, et al. Progressive decline of glucocerebrosidase in aging and Parkinson's disease. Ann Clin Transl Neurol. 2015;2(4):433-438.

13. Burbulla LF, et al. A modulator of wild-type glucocerebrosidase improves pathogenic phenotypes in dopaminergic neuronal models of Parkinson's disease. Sci Transl Med. 2019;11(514):eaau6870.

14. Patnaik S, et al. Discovery, structure-activity relationship, and biological evaluation of noninhibitory small molecule chaperones of glucocerebrosidase. J Med Chem. 2012;55(12):5734-5748.

15. Weintraub D, et al. Patterns and trends in antipsychotic prescribing for Parkinson disease psychosis. Arch Neurol. 2011;68(7):899-904

16. Hall MD, et al. Fluorescence polarization assays in high-throughput screening and drug discovery: a review. Methods Appl Fluoresc. 2016;4(2):022001.

17. Rossi AM, Taylor CW. Analysis of protein-ligand interactions by fluorescence polarization. Nat Protoc. 2011;6(3):365-387.

18. Ysselstein D, et al. LRRK2 kinase activity regulates lysosomal glucocerebrosidase in neurons derived from Parkinson's disease patients. Nat Commun. 2019;10(1):5570.

19. Sardi SP, et al. CNS expression of glucocerebrosidase corrects alpha-synuclein pathology and memory in a mouse model of Gaucher-related synucleinopathy. Proc Natl Acad Sci U S A. 2011;108(29):12101-12106.

20. Riedel M, et al. Quetiapine in the treatment of schizophrenia and related disorders. Neuropsychiatr Dis Treat. 2007;3(2):219-235.

21. Hashimoto $\mathrm{N}$, et al. Long-term efficacy and tolerability of quetiapine in patients with schizophrenia who switched from other antipsychotics because of inadequate therapeutic response-a prospective open-label study. Ann Gen Psychiatry. 2015;14(1):1.

22. Brundin P, Wyse RK. The Linked Clinical Trials initiative (LCT) for Parkinson's disease. Eur J Neurosci. 2019;49(3):307-315.

23. Pushpakom S, et al. Drug repurposing: progress, challenges and recommendations. Nat Rev Drug Discov. 2019;18(1):41-58.

24. Zheng J, et al. Design and synthesis of potent quinazolines as selective $\beta$-glucocerebrosidase modulators. J Med Chem. 2016;59(18):8508-8520.

25. Krainc D, et al. Substituted 4-methyl-pyrrolo[1,2-a]pyrimidine-8-carboxamide compounds and uses thereof for modulating glucocerebrosidase activity. https://patents.google.com/patent/US10040799B2/en?q=Preparation+of+4-methyl-pyrrolo[1\%2c2-a] pyrimidine-8-carboxamide+compounds+glucocerebrosidase +modulators.\&oq=+Preparation +of +4 -methyl-pyrrolo[1\%2c2-a] pyrimidine-8-carboxamide+compounds+as+glucocerebrosidase+modulators. + . Accessed August 27, 2021

26. Kriks S, et al. Dopamine neurons derived from human ES cells efficiently engraft in animal models of Parkinson's disease. Nature. 2011;480(7378):547-551.

27. Mazzulli JR, et al. Detection of free and protein-bound ortho-quinones by near-infrared fluorescence. Anal Chem. 2016;88(4):2399-2405. 